\title{
Selection and Applications of Automatic Spot Welding Methods
}

\author{
Shyamjith Uralath ${ }^{1}$,Hemant Raj Singh ${ }^{2}$ \\ Department of Mechanical Engineering, Manipal University Jaipur, India \\ Assistant Professor, Department of Mechanical Engineering, Manipal University Jaipur, India
}

\begin{abstract}
Spot Welding is one of the important applications of industrial robots. The speed, precision, efficiency and the resulting cost reductions due to mass production are well accepted and documented advantages of automation of spot welding process using robots compared to manual welding. The initial installation costs of the spot welding robot and its necessary accessories limit its application for large scale industries. The two methods of the spot welding process i.e. the spot welding gun mounted on the robot and part being stationary or the robot mounted part and stationary spot welding gun have unique applications depending on the complexity of the part as well as process. Instead of spot welding gun attached to the robot, a stationary spot welding gun and a moving robot carrying a part can be used for parts that require movements in four degrees of freedom. This paper discusses in detail about the selection and application of the two methods of spot welding.
\end{abstract}

Key Words: Automation, Industrial Robots, Spot Welding

\section{Introduction}

Automation is the use of control systems and information technologies to reduce the need for human work in production of goods and services. In industries automation is a step ahead of mechanization which provides machineries to replace the human labour, whereas automation generally implies the integration of machines to a self-governing system which greatly reduces the need for human sensory and mental requirements. Out of the automation techniques, the automation using robots is the most important one. Within the industries many operations which are repetitive like pick and place, painting, welding etc. have been successfully automated. The improved accuracy, the level of consistency achieved, adaptability to hazardous and dangerous work environments etc. are some of the advantages of robots which gives robots an upper hand over humans. One of the important applications of industrial robots is spot welding which is used in high production applications mostly in automotive industries where mass production is required. Spot welding is preferred in the automotive industries due to the complex welding profile of the parts and also due to the difficulty in handling heavy parts manually. Due to the cost of installation of robots and the spot welding equipment set up on the robot the application of spot welding robots is mainly limited to large scale industries. For the small and medium scale industries, in order to meet the new challenges of increased global competition, manufactures are forced to seek new technologies for the improved production and cost reduction. Such costcutting efforts can only be achieved by automating manual processes and also by modifying current automation solutions with newer and more economical technologies. This paper discusses two methods of selection and application of spot welding methods.

\section{Method-1: Spot Welding Gun Mounted On The Robot Arm}

The major applications of spot welding using a robot are accounted for the automotive industries around the world. The speed, precision, efficiency and the resulting cost reductions due to mass production are well accepted and documented advantages of spot welding using robots .In this method, a spot welding gun is mounted on the robot arm for performing the welding operations on a stationary part having complex profiles. The robotic arm attached with the spot welding gun can perform welding with greater flexibility and accuracy on complex weld profiles and in hazardous work environments where manual welding is difficult to perform.

\subsection{Significant Parts of Spot Welding Setup}

Some of the important parts of the spot welding robots are

2.1.1 Robot: The robots used for spot welding are articulate robots which are also called as six-axis robots. Articulate robots consist of three rigid members connected by two revolute joints and mounted on a rotary base. Its kinematic arrangement closely resembles that of a human arm. Apart from the three axes which are required to reach any point is space, the articulate robots have three more axes to fully control the orientation of the end of arm which helps the robot achieving maximum flexibility, reach over and under the object and also covers a large workspace relative to the volume of robots. The robots carry a spot welding gun and a power pack at the end of the arm which weigh more than $100 \mathrm{~kg}$. The robots also require a substantial loom to carry the services to 
the welder like power supply from the transformers, water and air for cooling purpose and also the information from the sensors. For maintaining the consistency in the quality of welds, tip dressing stations are also fitted in spot welding cells.

2.1.2 Spot Welding Gun: A welding gun having a pair of opposing welding electrodes either one of which is movable towards the other to apply a pressure onto a work and away from the other to release the pressure is attached with the arm of the robot. Robots need linear motion control to close and open the weld tips at precise position with exact pressure. Pneumatic actuators have been popularly used. But due to the problems of consistency, weld quality and speed they have been replaced by electric servo actuators. A servomotor is attached to the servo actuator for adjusting the pressure applied to the work through movement of the movable welding electrode. It provides a better welding performance and quality, which is made possible by accurate control of position and torque. Control instruction send by the servo controller, which is part of the robot controller, drives the servo motor at a velocity and torque predefined by robotic programs.

2.1.4 Weld Controller: It consists of a welding current regulator which is used for adjusting the value of welding current applied through the opposite electrodes to the work and also a weld interval-timer for adjusting at least one weld interval and weld timing for the welding current and the pressure applied to the work.

2.1.5 The Spot Welding Swivel: It is a unit that permits passage of compressed air, cooling water, electric current and signals through different channels within a single rotating unit. It improves the safety through reduction of air, electric and water lines.

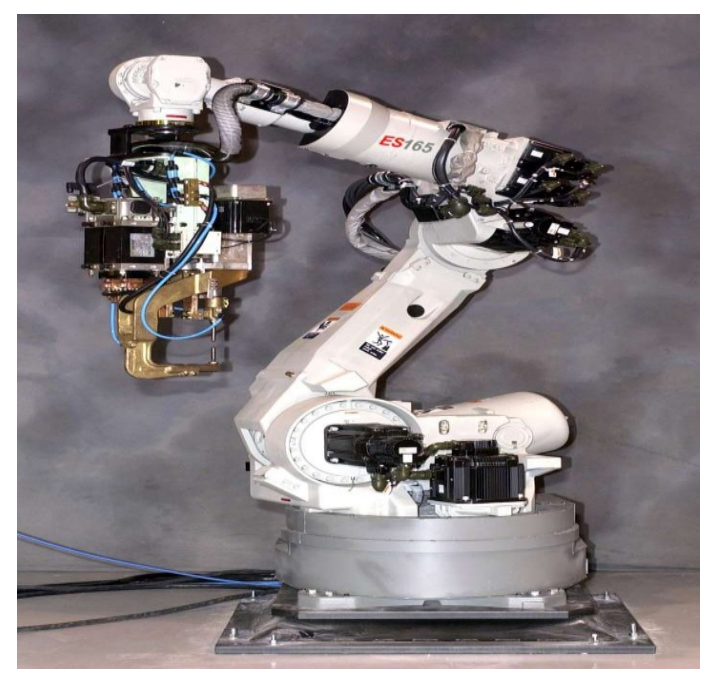

Fig 1: Spot Welding Gun Attached with a Robot

\subsection{Applications}

The important application of Robotic Spot Welding is in automotive industries. It is because the application of robots for spot welding is a good example of a cost-justified application that relieves humans from a tedious, difficult and hazardous job. Traditionally large and heavy body parts of the automobiles are held by clamping jigs, tacked together by operators using multiple welding guns, and the spot welded manually. To meet the requirement the odd shaped weld guns are to be moved and manoeuvred across complex welding profiles at high speeds. This process is time consuming and completely depends on operator efficiency. As the market has become more competitive globally, the manual spot welding process is replaced by robots which provide a much better quality product compared to the manual operation due to the repeatability and consistent positional accuracy.

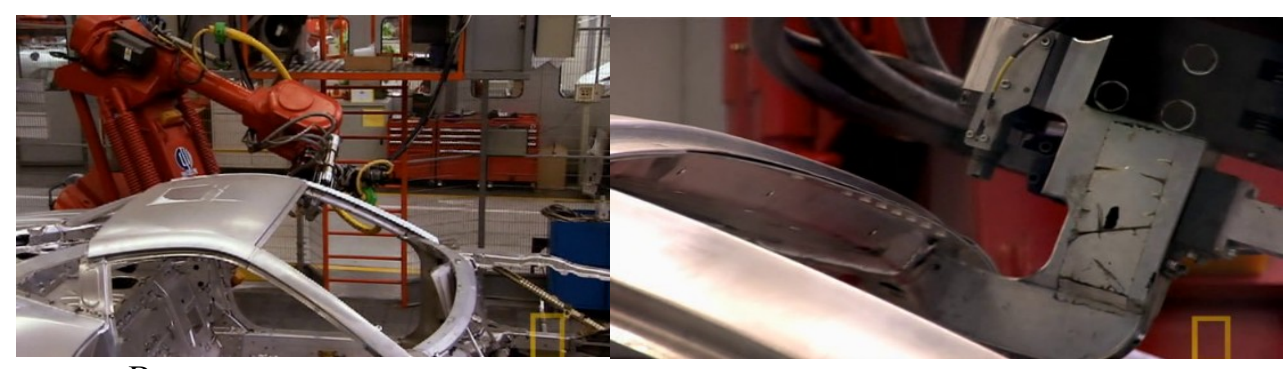

A

$\mathrm{B}$

Fig 2 A\& B: Robotic Spot Welding in Automotive Industry 


\subsection{Advantages}

a) The robots eliminate the workload of operators that is caused in handling heavy welding guns.

b) Improved quality is achieved through consistency and better repeatability in the location of the welds.

c) Improved safety by removing humans from a work environment where there are hazards from electrical shocks and burns.

d) Complex welding profiles can be welded easily where manual welding is difficult.

e) Improved productivity.

f) Cost effectiveness.

g) Less wasted material.

h) Good return on investment.

\section{Method-2: Stationary Spot Welding Gun}

The robots used for Spot Welding must have a certain capabilities and features to perform the welding process. The robots should be relatively large and it should have the sufficient payload capacity to hold welding gun and accessories associated with it. The robot should be capable of positioning and orienting the welding gun in places of the work-piece that must be difficult to access. Due to this reason, only robots with higher degree of freedom can be used for this purpose. Moreover the controller memory must have enough capacity to accomplish the positioning steps requires for spot welding cycle. Robots with higher degrees of freedom and more payload capacity to hold the spot welding gun and its accessories will increase the installation cost considerably. This makes the spot welding using a robot only preferable for mass production.

As discussed previously, in small scale and medium scale production the new challenges of increased global competition can be met by automating manual process and also by modifying current automatic solutions with newer and more economical technologies. In the case of spot welding, it is not economical to use a spot welding robot for making welds on simple and plane sheets where complex profile is not involved, at the same time the part has numerous weld points. The initial installation cost for the spot welding robot for this type of application will be very high compared to the previous method and also compared to the cost of part to be produced. For such parts, a spot welding method is used where the spot welding gun in stationary and the robot holds and moves the part to the desired weld position. This method possesses all the advantages of using a robot.

\subsection{Application}

The method of spot welding using a stationary spot welding machine and the robot holding and moving the part can be used for simple applications where no complex welding profiles are involved. In this method instead of operator carrying out the spot welding process, the robot moves the part to the stationary welding gun and welding is done at the desired programmed points. The method is explained with the following example.

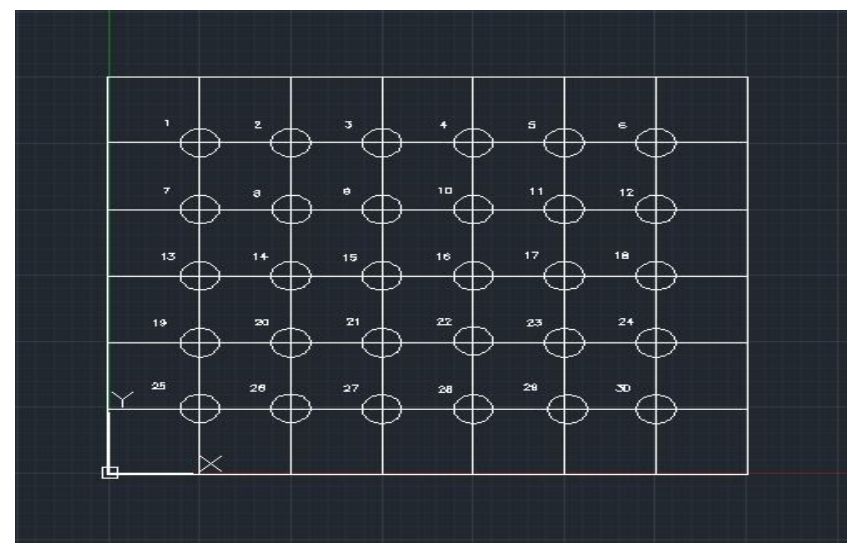

Fig 3: CAD Drawing of the Part

Figure-3 shows a plane sheet in which the weld points are marked for spot welding. Manual spot welding of this sheet will be time consuming and it is a complete labour oriented process. It completely depends on the skill of the operator doing the job. Robotic spot welding may be not be economical for such application considering the cost of installation. The best method to carry out such welding process is fixing the part to the arm of robot and the robot moving the part to the stationary welding gun where welding is carried out at the desired weld points as programmed. 


\subsection{Significant Parts of Welding using a Stationary Welding gun}

Apart from the welding setup of a spot welding robot this method is simple and consists of very few parts. The important parts are:

3.2.1 Robot: This application requires the movement of the robot $\mathrm{X}$ axis, $\mathrm{Y}$ axis and sometimes a rotation of the part depending upon the size and the weld points of the part. For the spot welding robot it is necessary to have high degrees of freedom for completing the complex weld profiles. But in this case a SCARA (Selective Compliance Assembly Robot Arm) robot which is a 4-axis robot can also be used. SCARA robots move in X, Y and $\mathrm{Z}$ plane like Cartesian robots, but it incorporates a theta axis at the end of the $\mathrm{Z}$ plane to rotate the end of arm tool. The only function of the robot used for this particular process is to move the part to the stationary welding gun and it involves only $\mathrm{X}$ axis and $\mathrm{Y}$ axis motion. Some applications may initially require a $\mathrm{Z}$ axis movement for carrying the part. Hence, for this application either a 4-axis SCARA robot or a 6-axis articulate robot is suitable.

3.2.2 Spot Welding Equipment: The spot welding equipment are composed basically with an electrical circuit which provides welding current, a control circuit that regulates welding current and welding time, and a mechanical system used to apply welding forces. The electrodes used should have high electrical and thermal conductivities and must develop low electric contact resistance in order to prevent deterioration of the workpiece and electrodes. The welding equipment also should be interfaced to the robot controller so that it can be programmed.

In this method the part is attached to the arm of the robot using a gripper so that it can be moved by the robot. Next step involves programming the robot and teaching the position of all the weld points to robots. The robot is moved to each and every weld point of the work-piece and then the co-ordinates of the weld points are taught to the robot using a teach pendant. The robot will be moving in the path exactly as it is taught. Robot moves to the weld position and the stationary welding gun will carry out the welding at the locations where it is taught.

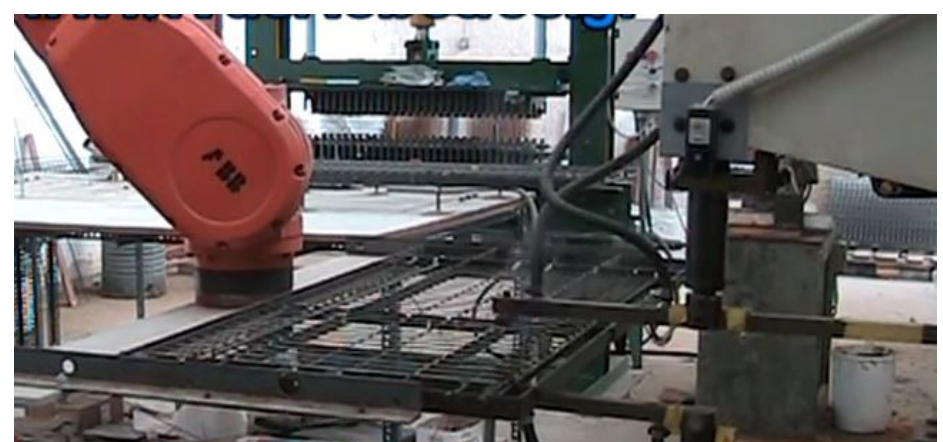

Fig 4: Welding with a Stationary Spot Welding Gun and Robot Moving PartCourtesy: VasRobotics

\subsection{Advantages}

a) The cost of installation is very less compared to that of the spot welding gun mounted to the robot.

b) The mass of the work-piece that the robot has to hold is considerably less compared to the spot welding gun mounted on the robot. Due to this reason robots with lower payload and lesser degree of freedom can be used for this application.

c) The largest source of failure in robot spot welding is the welding gun and the cables (robot dress) used to operate the gun. The dynamic motion of the robot causes fatigue to the cables which can cause damage for the welding gun through a crash. By using a stationary welding gun the robot dress is minimized and it make more robust application and more cell up time.

d) Increased operator safety.

\section{Comparison Between Two Methods}

\begin{tabular}{|l|l|l|}
\hline & \multicolumn{1}{|c|}{ Spot Welding Gun mounted on the Robot } & \multicolumn{1}{c|}{ Stationary Spot Welding gun and Movable Part } \\
\hline Cost & $\begin{array}{l}\text { The cost of installation of the robot and its } \\
\text { accessories is very high }\end{array}$ & The cost of installation is comparatively less. \\
\hline Robot & $\begin{array}{l}\text { Robots with higher degree of freedom can only be } \\
\text { used. That is 6-axis articulate robots. }\end{array}$ & $\begin{array}{l}\text { 4-axis robots can also be used as it involve only X-axis } \\
\text { and Y-axis motion??? }\end{array}$ \\
\hline $\begin{array}{l}\text { Complexity of } \\
\text { the welding } \\
\text { arrangement }\end{array}$ & $\begin{array}{l}\text { The welding arrangement is very complex with the } \\
\text { cables and hoses running around the robot which are } \\
\text { the main reasons for robot failure. }\end{array}$ & $\begin{array}{l}\text { As the robot is only holding the part the welding } \\
\text { arrangement is simple. }\end{array}$ \\
\hline Work-space & $\begin{array}{l}\text { A large work-space is required for the operation of } \\
\text { the robot and the spot welding gun }\end{array}$ & Work-space required is comparatively small. \\
\hline Application & $\begin{array}{l}\text { Application is mainly in automobiles industries } \\
\text { where mass production is required for complex } \\
\text { welding profiles. }\end{array}$ & $\begin{array}{l}\text { It can be used only for simple applications. Complex } \\
\text { welding profile cannot be welded with this approach }\end{array}$ \\
\hline
\end{tabular}




\section{Conclusion}

Spot welding using a welding gun mounted on the robot arm is widely used for large scale production mainly in automotive industries where it is difficult to carry out manual welding due to the complexity of the weld profiles and the huge parts. The cost of installation of the robots with higher degrees of freedom, with its necessary accessories and also its complex application makes it only suitable for mass production where the initial capital cost and running cost must be balanced over the life of the product. In the other method, where a stationary spot welding gun is used and the robot is moving the part, the cost of installation is reduced considerably. A 4-axis robot also can be used to perform this application which makes it suitable for simple welding application. It will have all the advantages of using a robot such as increased quality and better consistency etc. This method can only be used for simple application which does not involve any complex welding parts such asin the automotive industries.

\section{References}

[1] S. Arora and A. Gupta, Industrial automation and Robotics, Laxmi Publication Pvt Limited, 2009.

[2] M. P. Groover and M. Weiss, Industrial Robotics-Technology, Programming and Applications, McGraw-Hill International.

[3] J. Magar and R. Shelkikar, "Implementation of Robots in Spot Welding Process," IOSR Journal of Electronics and Communication Engineering, vol. 5, no. 3, April 2013.

[4] P. Singh, A. Kumar and M. Vashisth, "Design of a Robotic Arm with Gripper \& End effectors for Spot Welding," Universal Journal of Mechanical Engineering, pp. 92-97, 2013.

[5] S. R. Deb, Robotics technology and Flexible automation, Mc Graw Hill, 2004.

[6] R. Mittal and I. Nagrath, Robotics and Control, Tata McGraw Hills, 2003.

[7] www.globalrobots.com

[8] www.info.tolomatic.com 